\title{
Field of Stresses in an Isotropic Plane with Circular Inclusion under Tensile Stress
}

\author{
Yevgenii Yevgen'evich Deryugin ${ }^{1 \#}$, Galina Vasil'evna Lasko ${ }^{2}$ \\ ${ }^{1}$ Institute for Strength Physics and Materials Science (ISPMS SB RAS), Tomsk, Russia \\ ${ }^{2}$ Staatliche Materialprufungsanstalt (MPA), University of Stuttgart, Stuttgart, Germany \\ Email: "dee@ispms.tsc.ru
}

Received June 6, 2012; revised July 6, 2012; accepted July 18, 2012

\begin{abstract}
Within the framework of the linear theory of elasticity, the analytical equations for the components of the stress tensor for a plane with a circular inclusion under tensile loading have been derived using the method of superposition. The given approach allows one to describe the plane-stress state of the plane both for the case of rigid and "soft" inclusions.
\end{abstract}

Keywords: Linear Theory of Elasticity; Method of Superposition; Boundary Conditions; Stress Field Components; Inclusion; Circular Hole

\section{Introduction}

The presence of a material with other elastic characteristics in the local region of a solid under loading causes a non-homogeneous field of stress, thus being a stress concentrator of corresponding scale. However, there is a lack of papers on analytical representation of stress fields in a continuous media with stress concentrators. The urgency of this issue is no cast some doubt $[1,2]$. The widely-applied method which allows the derivation of analytical expressions for the stress field in a continuous medium with the elements of structure is the superpositional method of linear theory of elasticity [3-6]. With the help of this method the derivation of the equation for all components of the stress field in a plane with a hard inclusion under loading is derived in the present paper. The plane-stress state is taken into consideration. Solution for the stress field in an elastic plane with an absolutely rigid circular inclusion is presented in [7]. The general solution for elastic plane with a circular inclusion has been obtained in this paper, using the superposition method, when there is a difference between the elastic modules of the plane and inclusion. The solution for the rigid inclusion is a special case of the common solution. The distinctive features of the stress fields for the "hard" and "soft" inclusions are described.

\section{Analytical Derivation of the Stress State of the Plane with a Round Inclusion}

The solution of a given task is connected with the defini-

\footnotetext{
*This work was supported by the Russian Foundation of Basic Research. Project No. 08-10-01182a.

${ }^{\#}$ Corresponding author.
}

tion of the boundary condition on the contour of the inclusion. Assume, that $E_{1}, v_{1}$ are correspondingly the Young modulus and Poisson's ratio of the plane and $E_{2}$, $v_{2}$ are the Young modulus and Poisson's ratio of the inclusion. The scheme of loading is represented in Figure 1(a). The tensile stress is directed along the $y$-axis. In the works of Eshelby [8,9] it was shown, that in the case of an elliptical inclusion, being oriented symmetrically with respect to the tensile axis, the stress field inside the inclusion is homogeneous with zero $\sigma_{x y}$ component. Hence, it is homogeneous also in the case of inclusions of round shape. Let us define the stress field inside the inclusion by the components $\sigma_{y}=k_{y} \sigma, \sigma_{x}=k_{x} \sigma$ and $\sigma_{x y}=0$, where $k_{y}$ and $k_{x}$ are the components which have to be defined.

Let us apply a superposition principle, which is valid in the approximation of linear theory of elasticity. According to this principle, the total solution of the boundary problem can be represented in the form of superposition of more simple solutions under the condition that the resulting boundary conditions remain the same. Shown in Figure 1 is a case, which doesn't break this condition. It reduces to the separation of a homogeneous solution (Figure 1(b)) from the general solution, which has the characteristics given below:

$$
\sigma_{y}=k_{y} \sigma, \sigma_{x}=k_{x} \sigma, \sigma_{x y}=0 .
$$

Without this it remains the solution for the plate under biaxial external load (Figure 1(c)) under the condition that stresses are equal to zero only inside the inclusion. Along the loading axis the tensile stress operates (1 $\left.k_{y}\right) \sigma$, and along the $x$-axis the stress $-k_{y} \sigma$. 


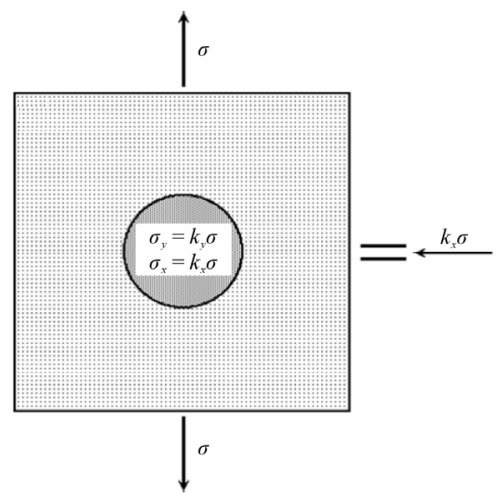

(a)

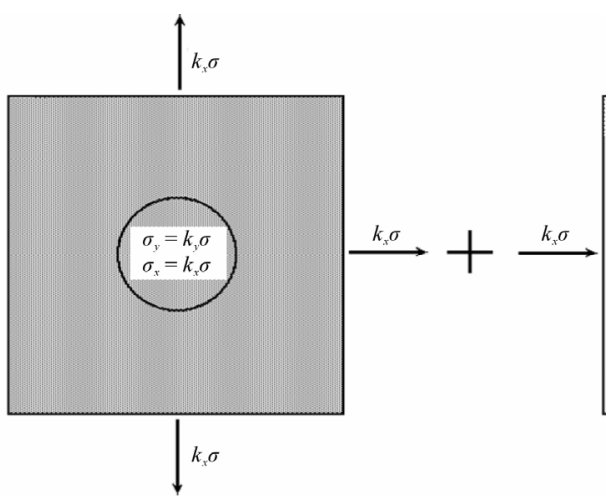

(b)

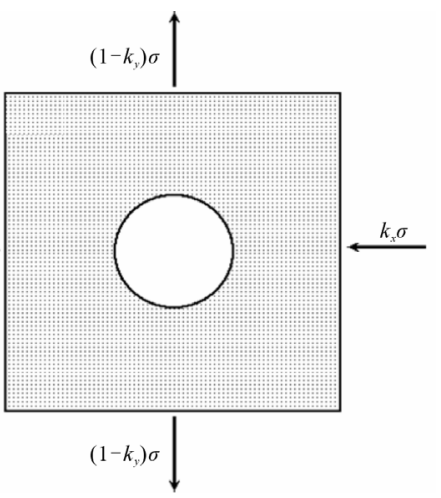

(c)

Figure 1. Schematic representation of the boundary conditions for the stress field of the plane with inclusion (a) in the form of the superposition of a homogeneous stress field (b), caused by biaxial external load, and the field of stresses of the plane under operation of biaxial external load (c), where the stresses are equal to zero only in the local region of the round shape.

Let us clarify the sense of the performed operation. From the total deformation of the inclusion (Figure 1(a)) we have subtracted the part, caused by the homogeneous stress field. According to Hooke's law, for the scheme in Figure 1(b) and stress field (1), it is homogeneous and characterized by the components

$$
\varepsilon_{y}=\left(k_{y}-k_{x} v_{2}\right) \sigma / E_{1}, \varepsilon_{x}=\left(k_{x}-k_{y} v_{1}\right) \sigma / E_{1}, \varepsilon_{x y}=0 .
$$

The elastic characteristics of materials of plane and inclusion are different. Naturally, there is a definite deformation, which together with deformation (2) defines the true deformation of the inclusion. This deformation, according to the scheme in Figure 1(c) defines the change in the shape of local region, in which the stresses are equal to zero, while along the $y$-axis the external tensile stress $\left(1-k_{y}\right) \sigma$ operates and along the $x$-axis - the external stress $-k_{x} \sigma$ : the field of point displacements inside the circular region in Figure 1(c) can be represented as caused by the deformation of inclusion with elastic characteristics tending to zero. It is seen that elastic displacements of the points inside the inclusion with the characteristics $E_{2}$ and $v_{2}$, and hence the boundary conditions on the contour of the inclusion will not change, if the displacements in the homogeneous stress field (1) are added inside the circle the displacements of the points of fictitious inclusion with the characteristics $E_{2} \rightarrow 0$ and $v_{2}$ $\rightarrow 0$ in the given plane under operation of the stress ( $1-$ $k_{y}$ ); along the $y$-axis and the stress $-k_{x} \sigma$ along the $x$-axis. In such a case, the absence of stresses in the round region doesn't mean the absence of the deformed material.

The deformation of the round region in Figure 1(c) it is not hard to define, knowing the displacements of the round region under the operation of the known boundary conditions. Shown in Figures 2(a)-(c) is the scheme of superposition of two separate solutions for uniaxial loadings, ensuring the pointed boundary conditions on the boundary of the circle, being equivalent to those for the plane with a circular hole under operation of uniaxial loading. In this connection, we can use the known solution of Kirsch [10].

For the case of the plane with the origin of coordinates at the center of the circular hole (in our case at the center of the inclusion with the characteristics $E_{2} \rightarrow 0$ and $v_{2} \rightarrow$ 0 ) under tension, the Kirsch problem defines the stress field beyond the round contour and displacement of the points of the contour itself. Usually, analytical expressions for the given characteristics are given in the polar coordinate system [3]. Transferring to the right-angle Cartesian coordinate system, for the boundary condition in Figure 2(b), the components of the stress field beyond the round circle will be characterized by the components (Appendix 1.1).

$$
\begin{aligned}
& \sigma_{y}^{*}=\sigma+\frac{\left(1-k_{1}\right) \sigma R^{2}}{2 r^{2}}\left[1+\frac{3 R^{2}+10 y^{2}}{r^{2}}-F+G\right], \\
& \sigma_{x}^{*}=\frac{\left(1-k_{1}\right) \sigma R^{2}}{2 r^{2}}\left[3-\frac{3 R^{2}+18 y^{2}}{r^{2}}+F-G\right], \\
& \sigma_{x y}^{*}=\frac{\left(1-k_{1}\right) \sigma R^{2} y x}{r^{4}}\left[3-\frac{2\left(3 R^{2}+4 y^{2}\right)}{r^{2}}+\frac{12 R^{2} y^{2}}{r^{4}}\right],
\end{aligned}
$$

where $R$ is the radius of the inclusion, $r^{2}=x^{2}+y^{2}$ is the distance from the center of the inclusion to the point with the coordinates $(x, y), F=8 y^{2}\left(3 a^{2}+2 y^{2}\right) / r_{4}$, $G=24 R^{2} y^{4} / r^{6}$.

For the boundary condition in Figure 2(c) we have

$$
\begin{aligned}
& \sigma_{y}^{* *}=\frac{-k_{2} \sigma R^{2}}{2 r^{2}}\left[1-\frac{3 R^{2}+14 y^{2}}{r^{2}}+F-G\right], \\
& \sigma_{x}^{* *}=\frac{k_{2} \sigma R^{2}}{2 r^{2}}\left[5-\frac{3 R^{2}+22 y^{2}}{r^{2}}+F-G\right], \\
& \sigma_{x y}^{* *}=\frac{-k_{2} \sigma R^{2} y x}{r^{4}}\left[5-\frac{2\left(3 R^{2}+4 y^{2}\right)}{r^{2}}+\frac{12 R^{2} y^{2}}{r^{4}}\right] .
\end{aligned}
$$




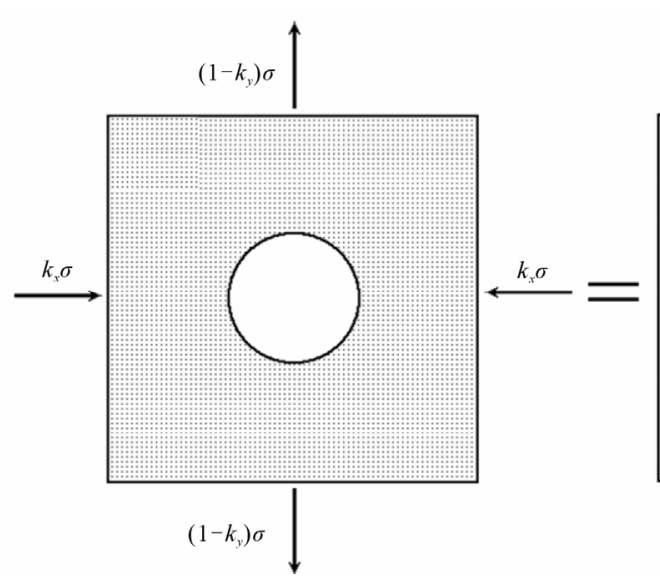

(a)

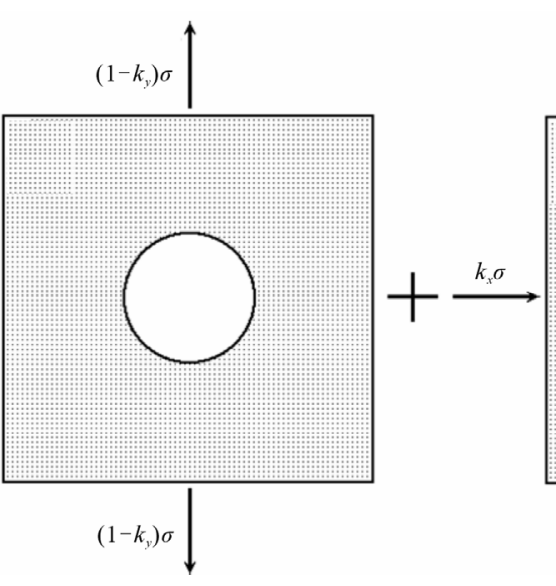

(b)

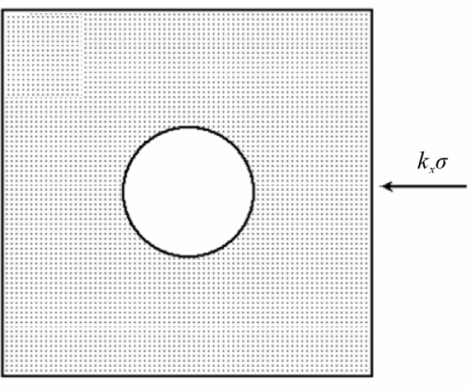

(c)

Figure 2. Schematical representation of the boundary condition for the field of stresses of the plane under operation of biaxial external load (a) in the form of superposition of the corresponding conditions for uniaxial loads (b, c), when in the local region of the round shape the stresses are equal to zero.

The superposition of the solution (3) and (4) together with the homogeneous stress field (1) $\left(\sigma_{y}^{0}, \sigma_{x}^{0}\right)$ defines the actual stress field beyond the inclusion.

$$
\begin{aligned}
& \sigma_{y}=\sigma_{y}^{*}+\sigma_{y}^{* *}+\sigma_{y}^{0}, \sigma_{x}=\sigma_{x}^{*}+\sigma_{x}^{* *}+\sigma_{x}^{0} \\
& \text { and } \sigma_{x y}=\sigma_{x y}^{*}+\sigma_{x y}^{* *},
\end{aligned}
$$

The displacements components of an arbitrary point $\left(x_{0}, y_{0}\right)$ on the boundary of the inclusion, corresponding to the boundary conditions in Figures 2(b) and (c) are defined by the equations:

$$
\begin{aligned}
& u_{y}^{*}\left(x_{0}, y_{0}\right)=3\left(1-k_{y}\right) y_{0} \sigma / E_{1}, \\
& u_{x}^{*}\left(x_{0}, y_{0}\right)=-\left(1-k_{y}\right) x_{0} \sigma / E_{1}, \\
& u_{y}^{* *}\left(x_{0}, y_{0}\right)=k_{x} y_{0} \sigma / E_{1}, \\
& u_{x}^{* *}\left(x_{0}, y_{0}\right)=-3 k_{x} x_{0} \sigma / E_{1} .
\end{aligned}
$$

The displacement components $u_{y}^{0}, u_{x}^{0}$ of an arbitrary point $\left(x_{0}, y_{0}\right)$ in the homogeneous stress field are defined by the corresponding homogeneous field of deformation (Appendix 1.2):

$$
\begin{aligned}
& u_{y}^{0}\left(x_{0}, y_{0}\right)=y_{0}\left(k_{y}-k_{x} n_{1}\right) \sigma / E_{1}, \\
& u_{x}^{0}\left(x_{0}, y_{0}\right)=x_{0}\left(k_{x}-k_{y} n_{1}\right) \sigma / E_{1} .
\end{aligned}
$$

By summation of the corresponding components in Equations (6) and (7), we obtain the components of the actual (real) displacements of an arbitrary point $\left(x_{0}, y_{0}\right)$ on the boundary of the inclusion.

$$
\begin{aligned}
& u_{x}\left(x_{0}, y_{0}\right)=u_{x}^{0}\left(x_{0}, y_{0}\right)+u_{x}^{*}\left(x_{0}, y_{0}\right)+u_{x}^{* *}\left(x_{0}, y_{0}\right) ; \\
& u_{y}\left(x_{0}, y_{0}\right)=u_{y}^{0}\left(x_{0}, y_{0}\right)+u_{y}^{*}\left(x_{0}, y_{0}\right)+u_{y}^{* *}\left(x_{0}, y_{0}\right) .
\end{aligned}
$$

It is easy to check that the given boundary conditions in displacements (8) satisfy the homogeneous field of deformation, characterized by the components:

$$
\begin{aligned}
& e_{x}=-\left[1+2 k_{x}-k_{y}\left(1-v_{1}\right)\right] \sigma / E_{1}, \\
& e_{y}=\left[3-2 k_{y}+k_{x}\left(1-v_{1}\right)\right] \sigma / E_{1}, \\
& e_{x y}=0,
\end{aligned}
$$

where there are two unknown coefficients $k_{y}$ and $k_{x}$. In (9) the deformation of the inclusion is expressed by the elastic characteristics of the plane. Due to the linearity of elastic deformation the solution (9) is unique.

On the other hand, accounting for the elastic properties of the inclusion itself, the stress field (1) in the inclusion (Figure 1(a)) corresponds to the homogeneous deformation, characterized by the components (2). Equating corresponding components in Equations (9) and (2) a system of two equations can be contained with two unknowns $k_{y}$ and $k_{x}$ which can be written in the following form:

$$
\begin{aligned}
& k_{y}\left(E_{1}+2 E_{2}\right)-k_{x}\left[v_{2} E_{1}+\left(1-v_{1}\right) E_{2}\right]=3 E_{2}, \\
& k_{x}\left(E_{1}+2 E_{2}\right)-k_{y}\left[v_{2} E_{1}+\left(1-v_{1}\right) E_{2}\right]=-E_{2} .
\end{aligned}
$$

Having solved the system, we shall find the values of unknown coefficients:

$$
k_{y}=\frac{E_{2}\left[\left(3-v_{2}\right) E_{1}+\left(5+v_{1}\right) E_{2}\right]}{\left(E_{1}+2 E_{2}\right)^{2}-\left[v_{2} E_{1}+\left(1-v_{1}\right) E_{2}\right]^{2}}
$$

and

$$
k_{x}=\frac{E_{2}\left[\left(3 v_{2}-1\right) E_{1}+\left(1-3 v_{1}\right) E_{2}\right]}{\left(E_{1}+2 E_{2}\right)^{2}-\left[v_{2} E_{1}+\left(1-v_{1}\right) E_{2}\right]^{2}} .
$$

\section{Results and Discussion}

Substituting the values $k_{y}$ and $k_{x}$ into Equations (1)-(5), all the necessary components of the stress field beyond 
the inclusion are obtained as

$$
\begin{aligned}
\frac{\sigma_{y}}{\sigma}= & 1+\frac{\left(1-k_{1}+k_{2}\right) R^{2}}{2 r^{2}}\left[1+\frac{3 R^{2}+10 y^{2}}{r^{2}}-F+G\right] \\
& -\frac{k_{2} R^{2}}{r^{2}}\left(1-\frac{2 y^{2}}{r^{2}}\right) ; \\
\frac{\sigma_{x}}{\sigma}= & \frac{\left(1-k_{1}+k_{2}\right) R^{2}}{2 r^{2}}\left[3-\frac{3 R^{2}+18 y^{2}}{r^{2}}-F+G\right] \\
& +\frac{k_{2} R^{2}}{r^{2}}\left(1-\frac{2 y^{2}}{r^{2}}\right) ; \\
\frac{\sigma_{x y}}{\sigma}= & \frac{\left(1-k_{1}-k_{2}\right) R^{2} y x}{r^{4}}\left[3-\frac{2\left(3 R^{2}+4 y^{2}\right)}{r^{2}}+\frac{12 R^{2} y^{2}}{r^{4}}\right] \\
& -\frac{2 k_{2} R^{2} y x}{r^{4}} ;
\end{aligned}
$$

Inside the inclusion, it is apparently, $\sigma_{y} / \sigma=k_{y}$, $\sigma_{x} / \sigma=k_{x}$ and $\sigma_{x y} / \sigma=0$.

Shown in Figure 3 are the distributions of the calculated components of the stress field for the case of inclusion $\mathrm{Al}_{2} \mathrm{O}_{3}\left(E_{2}=382 \mathrm{GPa}, v_{2}=0.3\right.$ [11]) in aluminium $\left(E_{1}=70 \mathrm{GPa}, v_{1}=0.3\right)$ under tension. It is seen that in the inclusion, the stress along the tensile axis is 1.4 times higher than the external (Figure 3(a)) applied stress. Along with it, near the inclusion, lowered stresses (b) and (c). On the boundary of the inclusion the components $\sigma_{x}$ and $\sigma_{x y}$ are characterized by significant positive and negative values in local zones.

Due to the large difference in the values of elastic modules the given case corresponds practically to the case of an absolutely rigid inclusion, for which the condition $E_{2} \rightarrow \infty, v_{2}=0$. Then from Equations (10) the coefficients $k_{y}$ and $k_{x}$ take the values

$$
k_{y}=\frac{5+v_{1}}{3+2 v_{1}-v_{1}^{2}} \text { and } k_{x}=\frac{1-3 v}{3+2 v_{1}-v_{1}^{2}} .
$$

It is seen from Equations (12), that in the plane-stress case the stresses from the absolutely rigid inclusion (11) do not depend on the elastic modulus $E_{1}$ of the surrounding matrix of material. The pattern of stress distribution qualitatively changes if $E_{2} \ll E_{1}$. Shown in Figure 4 is an example of $\sigma_{y}$ for the case being opposite to the previous one $\left(E_{1}=382 \mathrm{GPa}\right.$ and $\left.E_{2}=70 \mathrm{GPa}\right)$. That is practically case of the absolutely "soft" inclusion, when $k_{y}=$ $k_{x}=0$ refers. The solution turns out to be equivalent to the case of the plane with the circular cut-out under loading.

It is seen that the zones of elevated and lowered stresses changed places. The effect of the stress concentration in the given case is strongly pronounced.

Substituting $k_{y}$ and $k_{x}$ values in Equations (1)-(5), we obtain all the necessary components of the stress field beyond the inclusion.

\section{Conclusions}

The performed calculations show that in a number of cases, it is easy to obtain the solutions for the problems of the mathematical theory of elasticity by the superposition of known simpler solutions. So far it is sufficient to meet identical boundary conditions on the external and internal interfaces. In this paper the analytical equations describing in the plane-stress case the stress field in the plane sample with circular inclusion under tension have been derived. This stress field is shown to be represented in the form of the superposition of the homogeneous stress field (1) and the non-homogeneous stress field, being identical to the stress field of the plane with a round inclusion under biaxial loading. The latter consists of the stress arising under loading along the tensile axis, and being perpendicular to the tensile axis.

A.V. Mal has managed to derive the components of the stress field from the hard inclusion by selecting a definite stress function. In the monograph [7] these results are represented in the polar coordinate system. It is known that transformation of the components of the stress tensor under rotations and displacement is simple in the Cartesian coordinate system. The transition from the components of stress fields derived by Mal in the polar coordinate system, to the components $\sigma_{y}, \sigma_{x}$ and $\sigma_{x y}$ in the Cartesian coordinate system, results in very complicated expressions. Using the coefficients $k_{y}$ and $k_{x}(10)$ the expression for the components of the stress field take a simple form (11). It is easy to prove, that Mal's

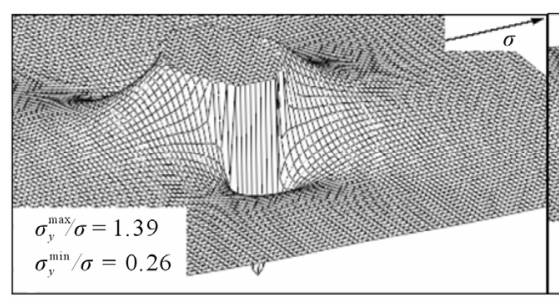

(a)

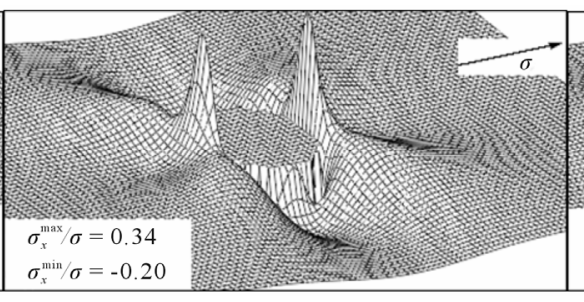

(b)

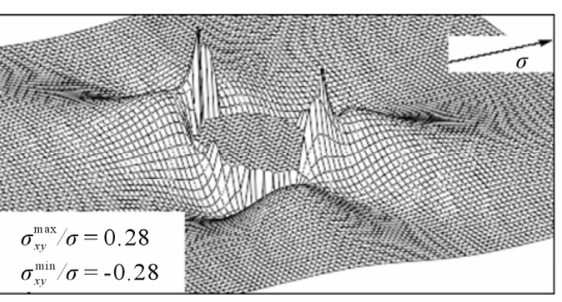

(c)

Figure 3. Spatial distribution of the stress field components in aluminum with a rigid circular $\mathrm{Al}_{2} \mathrm{O}_{3}$-inclusion. 


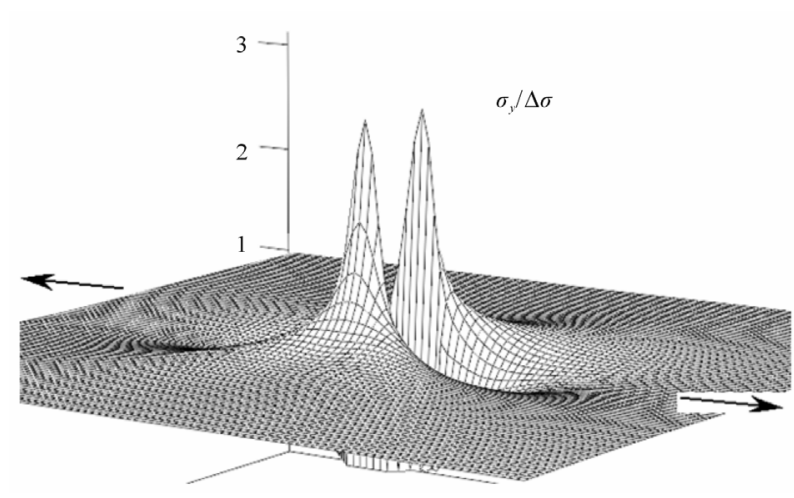

Figure 4. Spatial distribution of stress $\sigma_{y}$ in the plane with "soft" inclusion.

equations describe a homogeneous stress field (1) inside the inclusion. This fact testifies to the reliability of the obtained equations.

\section{REFERENCES}

[1] I. A. Ovid'ko and A. G. Sheinerman, "Elastic Fields of Nanoscopic Inclusions in Nanocomposites," Reviews on Advanced Materials Science, Vol. 9, 2005, pp. 17-33.

[2] N. A. Bert, A. L. Kolesnicova, A. E. Romanov and V. V. Tshaldushev, "Elastic Behavior of a Spherical Inclusion with a Given Uniaxial Dilatation," Physics of the Solid State, Vol. 44, No. 12, 2002, pp. 2139-2148. doi:10.1134/1.1529918
[3] M. Lai, E. Krempl and D. Ruben, "Introduction in Continuum Mechanics," 4th Edition, Elsevier, Oxford, 2010.

[4] S. P. Timoshenko and J. N. Goodier, "Theory of Elasticity," 3rd Edition, McGraw Hill, New York, 1970.

[5] V. A. Levin, "Many-Folded Superposition of Great Deformations in Elastic and Viscous-Elastic Bodies," Fizmatgiz, Moscow, 1999.

[6] S. L. Crouch and A. M. Starfield, "Boundary Element Methods in Solid Mechanics," George Allen \& Unwin, London, 1983.

[7] A. V. Mal and S. J. Singh, "Deformation of Elastic Solids," Prentice Hall, New York, 1992.

[8] D. E. Eshelby, "Definition of the Stress Field, Which Was Creating by Elliptical Inclusion," Proceedings of the Royal Society A, Vol. 241, No. 1226, 1957, p. 376. doi:10.1098/rspa.1957.0133

[9] D. E. Eshelby, "Elastic Field outside the Elliptical Inclusion," Proceedings of the Royal Society A, Vol. 252, No. 1271, 1959, p. 561. doi:10.1098/rspa.1959.0173

[10] G. Kirsch, "Die Theorie der Elastizitat und die Bedurfnisse der Festigkeitslehre," Zantralblatt Verlin Deutscher Ingenieure, Vol. 42, 1898, pp. 797-807.

[11] A. N. Babichev, N. A. Babushkina, A. M. Bratkovsky, et al., "Physical Values: Handbook," Energoatomizdat, Moscow, 1991. 


\section{Appendix}

\section{Kirsch's Solution in Cartesian Coordinate System}

\subsection{Calculation of Stress}

For the case of a plane under tensile stress $\sigma$ with the origin of the coordinates at the center of the circular hole (in our case at the center of the inclusion with the characteristics $E \rightarrow 0$ and $v \rightarrow 0$ ) Kirsch's problem defines the stress field beyond the circular contour and the displacement of the points of the contour themselves. The analytical equations for the stress tensor components are usually given in polar coordinate systems [2]. At an arbitrary point A (Figure I.1) with the radius-vector $r$ at the angle $\theta$ with respect to the tensile axis $0 y$ the stress tensor components are written in the form [1]:

$$
\begin{aligned}
& \sigma_{r}=\frac{\sigma}{2}\left(1-\frac{R^{2}}{r^{2}}\right)+\frac{\sigma}{2}\left(1+\frac{3 R^{4}}{r^{4}}-\frac{4 R^{2}}{r^{2}}\right) \cos 2 \theta ; \\
& \sigma_{\theta}=\frac{\sigma}{2}\left(1+\frac{R^{2}}{r^{2}}\right)-\frac{\sigma}{2}\left(1+\frac{3 R^{4}}{r^{4}}\right) \cos 2 \theta ; \\
& \sigma_{r \theta}=-\frac{\sigma}{2}\left(1-\frac{3 R^{4}}{r^{4}}+\frac{2 R^{2}}{r^{2}}\right) \cos 2 \theta .
\end{aligned}
$$

where $R$ is the radius of the circular contour, $r^{2}=x^{2}+y^{2}$ is the distance from the center of inclusion to point $\mathrm{A}$ with the coordinates $(x, y)$.

The transition to the Cartesian coordinate system is performed with the help of the famous equations:

$$
\begin{aligned}
& \sigma_{y}=\sigma_{r} \cos ^{2} \theta+\sigma_{\theta} \sin ^{2} \theta-\sigma_{r \theta} \sin 2 \theta ; \\
& \sigma_{x}=\sigma_{r} \sin ^{2} \theta+\sigma_{\theta} \cos ^{2} \theta+\sigma_{r \theta} \sin 2 \theta ; \\
& \sigma_{x y}=\left(\sigma_{r}-\sigma_{\theta}\right) \sin \theta \cos \theta+\sigma_{r \theta} \cos 2 \theta .
\end{aligned}
$$

Using the equations for the trigonometric functions

$$
\begin{gathered}
\cos \theta=\frac{y}{r}, \quad \sin \theta=\frac{x}{r}, \quad \cos 2 \theta=2 \frac{y^{2}}{r^{2}}-1, \\
\sin 2 \theta=2 \frac{x y}{r^{2}}, \quad \text { and } \sin ^{2} 2 \theta=4 \frac{y^{2}}{r^{2}}\left(1-\frac{y_{2}}{r^{2}}\right),
\end{gathered}
$$

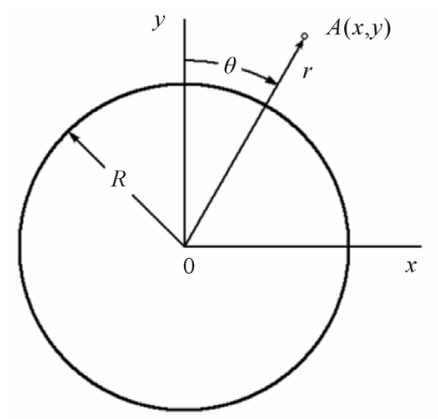

Figure I.1. Polar coordinate system in the plane with a circular hole. we obtain:

$$
\begin{aligned}
& \sigma_{y}=\sigma+\frac{\sigma R^{2}}{2 r^{2}}\left(1+\frac{3 R^{2}+10 y^{2}}{r^{2}}-F+G\right) ; \\
& \sigma_{x}=\frac{\sigma R^{2}}{2 r^{2}}\left(3-\frac{3 R^{2}+18 y^{2}}{r^{2}}+F-G\right) ; \\
& \sigma_{x y}=\frac{\sigma R^{2} x y}{2 r^{4}}\left(3-\frac{2\left(3 R^{2}+4 y^{2}\right)}{r^{2}}+\frac{12 R^{2} y^{2}}{r^{4}}\right),
\end{aligned}
$$

where $F=8 y^{2}\left(3 R^{2}+2 y^{2}\right) / r^{4}, G=24 R^{2} y^{4} / r^{6}$.

\subsection{The Calculation of Displacements of Inclusion Boundary}

The points displacements of the plane with circular zone free of stresses (the case is depicted in Figure 2(b)) under tension are defined by the known equations. In the case of plane-stress state in polar coordinates the displacement components are written in the form [9]:

$$
\begin{aligned}
& u_{r}= \\
& \frac{\sigma}{4 G r}\left\{(1-2 v) r^{2}+R^{2}+\left[4(1-v) R^{2}+r^{2}-\frac{R^{4}}{r^{2}}\right] \cos 2 \theta\right\} \\
& u_{\theta}=\frac{\sigma}{4 G r}\left[2(1-2 v) R^{2}+r^{2}+\frac{R^{4}}{r^{2}}\right] \sin 2 \theta .
\end{aligned}
$$

In particular, the displacements of the points of circular contour itself are equal:

$$
u_{r}=\frac{\sigma(1-v) R}{2 G}(1+2 \cos 2 \theta) ; u_{\theta}=\frac{\sigma(1-v) R}{G} \sin 2 \theta ;
$$

Here, $G$ is the shear modulus, and $v$ is Poisson's ratio of the plane.

The transition to Cartesian coordinates is realized with the help of equations

$$
u_{y}=u_{\theta} \cos \theta+u_{r} \sin \theta, u_{x}=u_{r} \cos \theta-u_{q} \sin \theta
$$

and for the point $\left(x_{0}, y_{0}\right)$ on the contour of the circle the above equations become very simple:

$$
u_{y}\left(x_{0}, y_{0}\right)=3 y_{0} \sigma / E_{1}, u_{x}\left(x_{0}, y_{0}\right)=-x_{0} \sigma / E_{1}
$$

Taking this into account, the displacements of an arbitrary point $\left(x_{0}, y_{0}\right)$ on the boundary of the inclusion, corresponding to the boundary conditions in Figures 2(b) and (c), are defined by the equations

$$
\begin{aligned}
& u_{y}^{0}\left(x_{0}, y_{0}\right)=y_{0}\left(k_{y}-v_{1} k_{x}\right) \sigma / E_{1}, \\
& u_{x}^{0}\left(x_{0}, y_{0}\right)=x_{0}\left(k_{x}-v_{1} k_{y}\right) \sigma / E_{1} \\
& u_{y}^{* *}\left(x_{0}, y_{0}\right)=k_{x} y_{0} \sigma / E_{1}, \\
& u_{x}^{* *}\left(x_{0}, y_{0}\right)=-3 k_{x} x_{0} \sigma / E_{1} .
\end{aligned}
$$


The displacement components $u_{y}^{0}$ and $u_{x}^{0}$ of the arbitrary point $\left(x_{0}, y_{0}\right)$ in the homogeneous stress field (1) are defined by the homogeneous field of deformation:

$$
\begin{aligned}
& u_{y}^{0}\left(x_{0}, y_{0}\right)=y_{0}\left(k_{y}-v_{1} k_{x}\right) \sigma / E_{1}, \\
& u_{x}^{0}\left(x_{0}, y_{0}\right)=x_{0}\left(k_{x}-v_{1} k_{y}\right) \sigma / E_{1}
\end{aligned}
$$

By summing of the corresponding components in Equations (I.3) and (I.4), we obtain the components of rea1 displacements of the arbitrary point $\left(x_{0}, y_{0}\right)$ on the boundary of the inclusion:

$$
\begin{aligned}
& u_{x}=u_{x}^{0}+u_{x}^{*}+u_{x}^{* *}=-\left[1+2 k_{x}-k_{y}\left(1-v_{1}\right)\right] x_{0} \sigma / E_{1} \\
& u_{y}=u_{y}^{0}+u_{y}^{*}+u_{y}^{* *}=\left[3-2 k_{y}+k_{x}\left(1-v_{1}\right)\right] y_{0} \sigma / E_{1} .
\end{aligned}
$$

The given boundaries conditions in displacements (I.6) are satisfied by the homogeneous field of deformation in the inclusion, characterized by the components:

$$
\begin{aligned}
& \varepsilon_{x}=-\left[1+2 k_{x}-k_{y}\left(1-v_{1}\right)\right] \sigma / E_{1} ; \\
& \varepsilon_{y}=\left[3-2 k_{y}+k_{x}\left(1-v_{1}\right)\right] \sigma / E_{1} .
\end{aligned}
$$

Due to linearity of the elastic deformation, the solution (1.7) is unique. 